\title{
Dynamics of localized and patterned structures in the Lugiato-Lefever equation determine the stability and shape of optical frequency combs
}

\author{
P. Parra-Rivas, ${ }^{1,2}$ D. Gomila, ${ }^{2}$ M. A. Matías, ${ }^{2}$ S. Coen,${ }^{3}$ and L. Gelens ${ }^{1, *}$ \\ ${ }^{1}$ Applied Physics Research Group, APHY, Vrije Universiteit Brussel, 1050 Brussels Belgium \\ ${ }^{2}$ Instituto de Física Interdisciplinar y Sistemas Complejos, IFISC (CSIC-UIB), Campus Universitat de les Illes Balears, \\ E-07122 Palma de Mallorca, Spain \\ ${ }^{3}$ Department of Physics, The University of Auckland, Private Bag 92019, Auckland 1142, New Zealand
}

(Received 23 October 2013; published 10 April 2014)

\begin{abstract}
It has been recently uncovered that coherent structures in microresonators such as cavity solitons and patterns are intimately related to Kerr frequency combs. In this work, we present a general analysis of the regions of existence and stability of cavity solitons and patterns in the Lugiato-Lefever equation, a mean-field model that finds applications in many different nonlinear optical cavities. We demonstrate that the rich dynamics and coexistence of multiple solutions in the Lugiato-Lefever equation are of key importance to understanding frequency comb generation. A detailed map of how and where to target stable Kerr frequency combs in the parameter space defined by the frequency detuning and the pump power is provided. Moreover, the work presented also includes the organization of various dynamical regimes in terms of bifurcation points of higher codimension in regions of parameter space that were previously unexplored in the Lugiato-Lefever equation. We discuss different dynamical instabilities such as oscillations and chaotic regimes.
\end{abstract}

DOI: 10.1103/PhysRevA.89.043813

PACS number(s): 42.65.Sf, 42.65.Tg, 05.45.-a, 47.54.-r

\section{INTRODUCTION}

Optical frequency combs can be used to measure light frequencies and time intervals more easily and precisely than ever before [1], opening a large avenue for applications. Traditional frequency combs are usually associated with trains of evenly spaced, very short pulses. More recently, a new generation of comb sources has been demonstrated in compact high- $Q$ optical microresonators with a Kerr nonlinearity pumped by continuous-wave laser light [2]. These combs are now referred to as Kerr frequency combs and have attracted a lot of interest in the last few years [3]. Interestingly, it has been demonstrated that Kerr frequency combs can be modeled in a way that is strongly reminiscent of temporal cavity solitons (CSs) in nonlinear cavities [4-6]. Temporal CSs have been experimentally studied in fiber resonators [7] and their description is based on a now classical equation, the Lugiato-Lefever equation (LLE), that describes pattern formation in optical systems [8].

Lugiato and Lefever, in 1987, introduced the mean-field model of nonlinear optical cavities, in which alternation of propagation around the cavity with coherent addition of an input field is replaced by a single partial differential equation with a driving term [8]. The LLE is applicable to different types of cavities, as shown in Fig. 1. It was originally derived to describe a ring cavity or a Fabry-Perot resonator with a transverse spatial extension and partially filled with a nonlinear medium [8] [see Figs. 1(a) and 1(b)]. The LLE can also be used in the context of single-mode fiber cavities [see Fig. 1(c)] [7]. In this case, the spatial coordinate in the LLE for a spatially extended cavity with diffraction is replaced by a time coordinate to model chromatic dispersion of light in the longitudinal (temporal) direction. Finally, more recently, the

\footnotetext{
*lendert.gelens@vub.ac.be
}

LLE has been applied to microresonators [see Fig. 1(d)] in the context of optical frequency combs [4-6,9].

The interplay between diffraction and/or dispersion and nonlinearity can lead to the formation of complex spatiotemporal structures inside the cavity, such as patterned and localized solutions [see Fig. 1(e)]. In the cavities presented in Figs. 1(a) and 1(b), a spatially localized bright light spot embedded in a homogeneous background of light has been shown to exist at the output of the resonator [10]. Such structures, localized in space, are also known as spatial cavity solitons (CSs). Similarly, in the cavities presented in Figs. 1(c) and 1(d), a stable structure that is localized in time can exist, also known as temporal cavity solitons [7]. A sketch of such a localized structure, both in the time (spatial) domain as well as in the corresponding Fourier domain, is shown in Fig. 1(e).

The formation and stability of patterns and CSs have been theoretically studied in great detail in the LLE, both in a one-dimensional (1D) and a two-dimensional (2D) setting $[8,11-15]$. The theory developed for CSs in the LLE was mainly motivated by the potential application of spatial CSs as information carriers in all-optical memories $[10,16]$. Recent experimental observations of 1D temporal CSs in fiber resonators [7] have renewed the interest in the LLE. This interest was further strengthened when it was demonstrated that the LLE can also be used to efficiently model Kerr frequency combs and that these were found, in some conditions, to be closely related to temporal CSs present inside the cavity [4-6]. Experimental evidence of CSs in microresonators has emerged shortly after [17]. The theory that has been developed for spatial CSs can thus potentially provide important information about the properties of Kerr frequency combs. In 1D, CSs have mainly been studied for lower values of the cavity frequency detuning, where they were shown to be always stable [13]. However, in the context of Kerr frequency combs, experiments are often carried out at relatively high pump power $[2,18]$. Because of the Kerr-induced tilt of the cavity resonance, the 


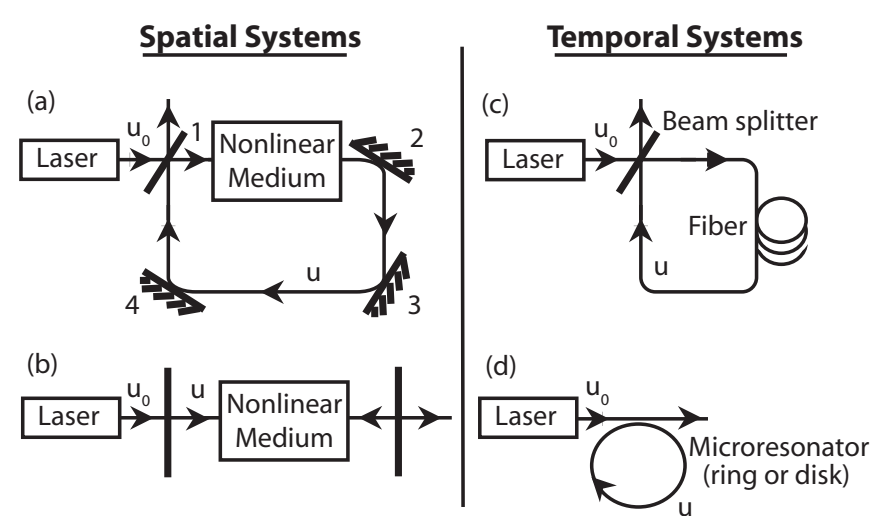

(e)
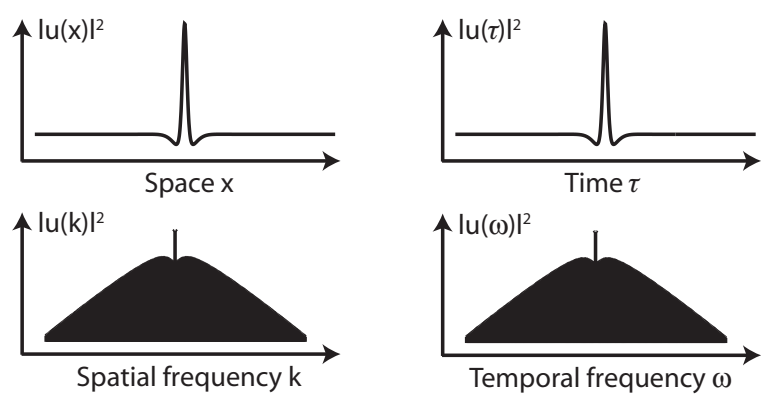

FIG. 1. Different setups where the mean field $u$ is described by the Lugiato-Lefever equation (LLE). (a) A ring cavity partially filled with a nonlinear medium. Mirror 1 partially transmits the input beam $u_{0}$, while mirrors 2-4 are completely reflecting. (b) A Fabry-Perot resonator filled with a nonlinear medium. (c) A nonlinear all-fiber cavity. (d) A microresonator where the cavity can be either a ring or a disk. (e) A sketch of a localized structure, both in the time (spatial) domain and in the corresponding Fourier domain.

detuning, which is measured from the center of the linear (cold) resonance, is correspondingly large. Only a few recent studies have been reported in this regime $[5,19,20]$. In particular, a fiber cavity experiment has demonstrated that CSs can display periodic oscillations, and preliminary numerical analysis has also revealed various chaotic regimes [20].

In this work, we aim to realize two goals: (i) to interpret how various coherent structures in microresonators, such as patterns and solitons, are intimately related to different types of Kerr frequency combs; (ii) to expand the study of the LLE to operating regimes that will prove to be of key importance for frequency comb generation, but so far have not been much explored. Such a detailed analysis of the unfolding of the rich dynamical behavior of the LLE for higher values of the detuning will also prove to be of fundamental interest for researchers working in the field of dissipative solitons.

The organization of this paper will be as follows. In Sec. II, we will introduce the Lugiato-Lefever model in more detail. In Sec. III, we will discuss the coexistence of multiple solutions, such as homogeneous solutions, patterned solutions, and localized solutions. We will show that all these structures are organized in a so-called snaking diagram and will discuss its importance in the context of frequency comb generation. In Sec. IV, we consider how the homogeneous state can connect to a patterned state and back, thus forming CSs. For transverse systems, this is referred to as the spatial dynamics and we will keep this terminology for combs for which the spatial coordinate is replaced by a longitudinal (temporal) variable. This study provides us with essential information about the location in parameter space where stable CSs and frequency combs can be found. In Sec. V, the dynamical behavior of single-peak CSs and their corresponding frequency combs are studied in the parameter space defined by the cavity detuning and the pump power. Finally, we draw our conclusions in the final Sec. VI.

\section{LUGIATO-LEFEVER EQUATION}

The LLE is a prototype model describing an optical cavity filled with a nonlinear Kerr medium as derived by Lugiato and Lefever in order to study pattern formation in this system [8]. Later studies have then demonstrated the existence of CSs in the LLE [11-15]. The LLE was originally obtained through a mean-field approximation, describing the dynamics of the slowly varying amplitude of the electromagnetic field $u(\varphi, t)$ in the paraxial limit, where $\varphi=x$ is the spatial coordinate transverse to the propagation direction. Here, we consider only one transverse dimension. The time evolution of the electric field over many cavity round trips can then be described as follows after a suitable rescaling of the variables [8]:

$$
\partial_{t} u=-(1+i \theta) u+i|u|^{2} u+u_{0}+i \partial_{\varphi}^{2} u .
$$

The first term on the right-hand side describes cavity losses (the system is dissipative by nature); $u_{0}$ is the amplitude of the homogeneous (plane-wave) input field or pump; $\theta$ measures the cavity frequency detuning between the frequency of the input pump and the nearest cavity resonance; $\partial_{\varphi}^{2}$ models diffraction; and the sign of the cubic term is set so that it corresponds to the self-focusing case. In the case of a fiber resonator or a whispering-gallery-mode (WGM) microresonator, the LLE still holds, but the input field $u_{0}$ is to be interpreted as the amplitude of the continuous-wave pump beam while the field $u(\varphi, t)$ is now a function of two time scales, with $\varphi=\tau$ a time coordinate (also known as fast time) in the frame moving with the group velocity $[4,5,7]$. The spatial derivatives are thus replaced by time derivatives $\partial_{\varphi}^{2} u=\partial_{\tau}^{2} u$, and the sign of this term is such that one has anomalous dispersion. Note that in our study here, we only consider second-order dispersion. Several works have also studied the influence of higher-order dispersion on the dynamics of dissipative structures in the LLE [21-23]. In the case of WGM microresonators, the LLE has also been shown to remain valid when replacing the coordinate $\varphi$ by the resonator's azimuthal angle [6].

The homogeneous steady-state (HSS) solutions $u_{s}$ of the LLE (1) are easily found by setting all derivatives to be zero:

$$
I_{s}\left[1+\left(\theta-I_{s}\right)^{2}\right]=I_{0} .
$$

The above equation is the classic cubic equation ( $\mathrm{S}$-shape response) of dispersive optical bistability, with $I_{s}=\left|u_{s}\right|^{2}$ and $I_{0}=\left|u_{0}\right|^{2}$. For $\theta<\sqrt{3}$, only one homogeneous solution exists, hence the system is monostable. In the context of spatial CSs, this monostable regime has been studied in great detail [8,11-15]. Much less analysis has been done when $\theta>\sqrt{3}$. In this case, for a range of input intensities $I_{0}$ [Eq. (2)] has three homogeneous solutions, out of which one is a saddle solution. Hence, the system is often called to be bistable. In this 

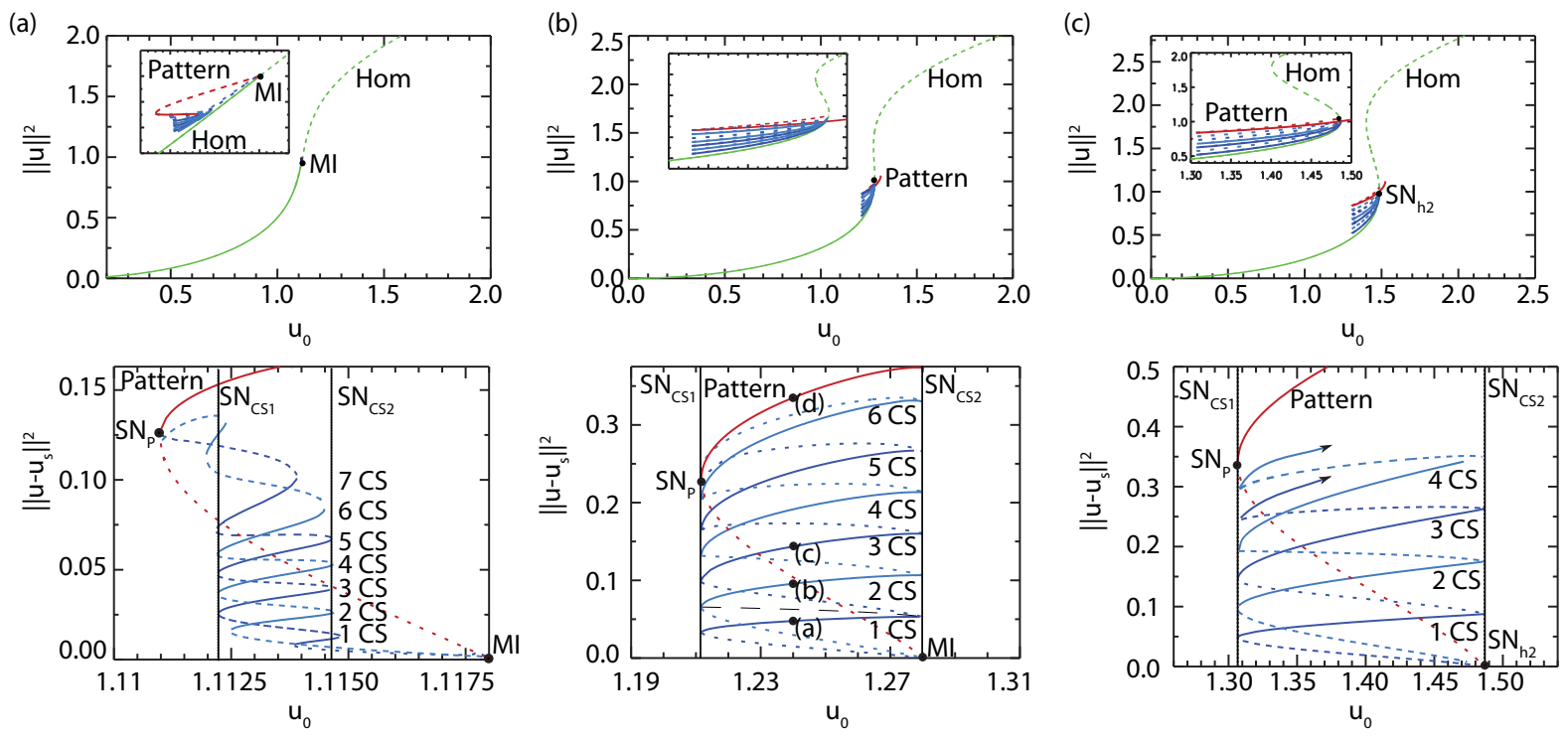

FIG. 2. (Color online) Various solution branches of the LLE as function of the pump $u_{0}$ for different values of the detuning $\theta[\theta=1.5$ (a), $\theta=1.8$ (b), $\theta=2.1$ (c)]. The (green) line denoted by "Hom" shows the homogeneous steady states (HSS) solutions of Eq. (1), where the inset shows a zoom of the region around the modulational instability (MI). A patterned branch (red curve, denoted "Pattern") is created subcritically in MI. The bottom panels show a more detailed picture of the snaking region where CSs (blue curve) exist, plotting the energy of the solution after removal of the homogeneous background. The snaking region is located between two other saddle-node bifurcations, called $\mathrm{SN}_{\mathrm{CS} 1,2}$. All solid (dotted) lines correspond to stable (unstable) solutions. The dots denoted by (a)-(d) in panel (b) correspond to solutions of which the profile is shown in Fig. 4.

bistable region, much more complex dynamics are observed in the LLE [20].

One can easily show the existence of two HSSs analytically by looking for the points where the derivative $\partial I_{0} / \partial I_{S}$ is equal to zero:

$$
\frac{\partial I_{0}}{\partial I_{s}}=1+\left(\theta-I_{s}\right)^{2}-2\left(\theta-I_{s}\right) I_{s}=0 .
$$

The solutions to this equation give the turning points of the bistable response, also known as the saddle-node ( $\mathrm{SN})$ bifurcations of the HSSs:

$$
I_{s}^{\mathrm{SN}_{h 1,2}}=\frac{2 \theta}{3} \pm \frac{1}{3} \sqrt{\theta^{2}-3} .
$$

It is clear that for $\theta^{2}<3$ there are no turning points [see Fig. 2(a)], while for $\theta^{2}>3$ there are two, $\mathrm{SN}_{h 1,2}$, and the system is bistable [see Figs. 2(b) and 2(c)]). Figures 2(a)-2(c) are plotted versus the input power for selected detunings while Figs. 3(a) and 3(b) present similar plots but as a function of detuning for selected pump powers. This second set of plots may be more relevant to the microresonator Kerr frequency comb context, in which the pump laser frequency is used as an important control parameter and for which thermal effects also strongly affect the detuning.

Analyzing the linear stability of the HSS to perturbations of the form $\exp (i k \varphi+\sigma t)$ [12], one finds that, for $\theta<\sqrt{3}$, the HSS loses its stability at $I_{s}=1$ with critical wave number $k_{c}=\sqrt{2-\theta}$ at which point a patterned solution is created either supercritically $(\theta<41 / 30)$ or subcritically $(\theta>41 / 30)$ [12]. In the bistable regime $(\theta>\sqrt{3})$, one can differentiate two different cases: $\sqrt{3}<\theta<2$, in which, as before, the MI occurs at $I_{s}=1$, and $\theta>2$, in which the critical wave number is zero and the HSS is stable all the

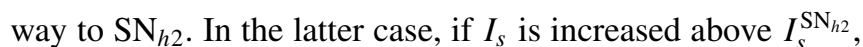
the HSS jumps to the upper branch, but the upper branch HSS has always a wide range of unstable wave numbers, whose growth leads to a spatiotemporal chaotic regime called optical turbulence [24,25].

In this work, we will show how localized and patterned solutions are organized in both the monostable and the bistable regimes, putting more emphasis on the bistable region as the monostable one has already been studied in depth. A typical example of the bifurcation diagram of the homogeneous solutions is shown in Figs. 2(a)-2(c) for values of the detuning $\theta$ in each characteristic region: $\theta<\sqrt{3}, \sqrt{3}<\theta<2$, and $\theta>2$. In the bistable region shown in Figs. 2(b) and 2(c), there exists a small region of input powers where the two homogeneous solutions coexist. The homogeneous solution with the highest intensity is always unstable in Eq. (1). A pattern is created subcritically at the modulational instability for $\theta<2$ such that a stable pattern can coexist with the stable homogeneous solution in a certain range of parameters. For $\theta>2$, although the system goes directly to optical turbulence above $\mathrm{SN}_{h 2}$, stable periodic patterns persist below threshold. Such coexistence is known to potentially give rise to localized structures, such as CSs, as will be discussed in the next section.

\section{CAVITY SOLITONS, PATTERNS, AND FREQUENCY COMBS}

The bottom panels in Figs. 2(a)-2(c), 3(a), and 3(b) show a zoom of the region where a subcritical branch of spatially periodic states (red) coexists with a stable homogeneous solution (green). For $\theta>41 / 30$, the pattern branch is unstable at its point of origin (MI), but acquires stability at finite 
(a)
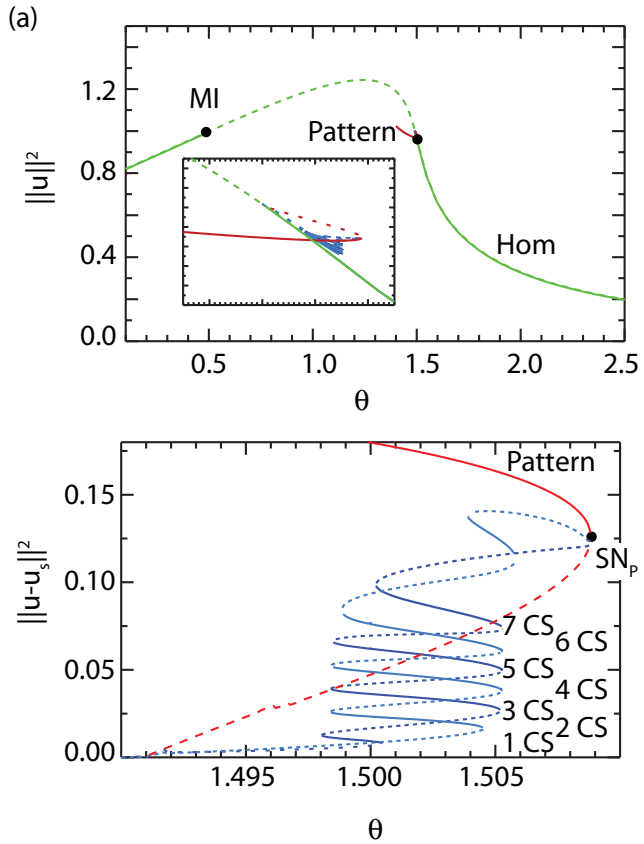

(b)
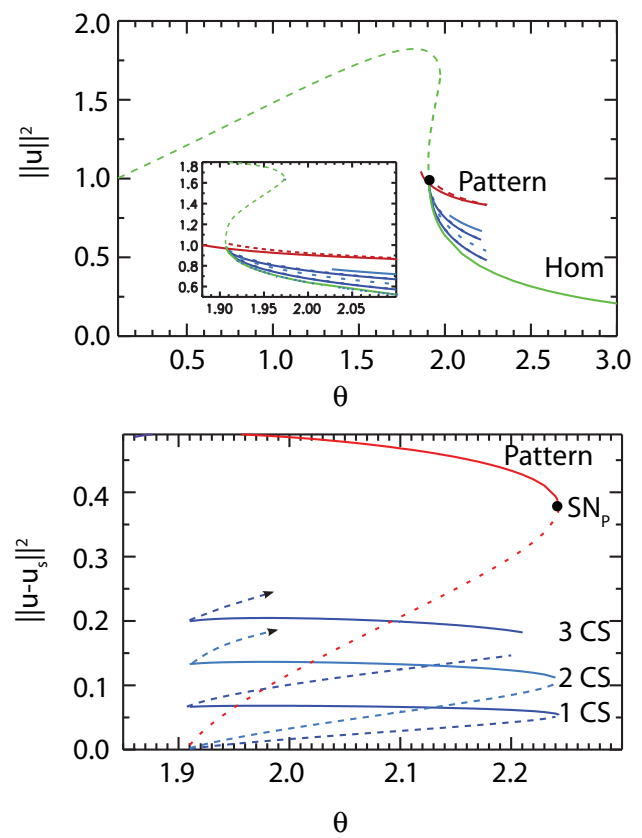

FIG. 3. (Color online) Same as Fig. 2 but as function of the detuning $\theta$ for different values of the pump $u_{0}\left[u_{0}=1.114\right.$ (a), $u_{0}=1.35$ (b)].

amplitude at a saddle-node bifurcation $\mathrm{SN}_{P}$. In addition, it is known that there are two branches of CSs (blue) that bifurcate from the homogeneous solution simultaneously with the periodic states, and do so likewise subcritically [13]. These states are therefore also initially unstable. When followed numerically, these states become better and better localized, and once their amplitude and width become comparable to the amplitude and wavelength of the periodic state, these CS states begin to grow in spatial extent by adding peaks symmetrically on either side, thereby forming bound states of CSs. In a bifurcation diagram, this growth is associated with back and forth oscillations across a pinning interval $\left[\mathrm{SN}_{\mathrm{CS} 1}-\mathrm{SN}_{\mathrm{CS} 2}\right]$. This behavior is known as homoclinic snaking [26-30], and is associated with repeated gain and loss of stability of the associated localized structures. This snaking structure has been experimentally verified for spatial CSs in a semiconductor-based optical system [31].

Figure 2 shows a clear difference in the location of the pinning interval $\left[\mathrm{SN}_{\mathrm{CS} 1}-\mathrm{SN}_{\mathrm{CS} 2}\right]$ when transitioning from the monostable [Fig. 2(a)] to the bistable region of operation [Figs. 2(b) and 2(c)]. For $\theta<\sqrt{3}$, the pinning interval where CSs can be found spans only a part of the region of coexistence between the stable pattern and the stable homogeneous solution, while for increasing values of the detuning in the bistable region $(\theta>\sqrt{3})$, the pinning interval quickly spans this entire region of coexistence. Another clear trend is that the CSs exist over a much broader range of values of the input amplitude $u_{0}$ and detuning $\theta$ as both $u_{0}$ and $\theta$ are increased in the bistable region. This evolution will be discussed in more detail in Sec. IV. We remark that for increasing values of the detuning $\theta$ it becomes increasingly difficult to numerically track the CS snaking branches. We believe this is not a numerical artifact, but rather that the snaking structure becomes more complex for $\theta>2$, hindering the numerical tracking of these CS branches (although solutions with multiple CSs seem to persist). Understanding the detailed snaking structure for high values of $\theta$ is beyond the scope of this paper and it will be addressed elsewhere.

In Fig. 4, we show typical profiles of the stable CSs that exist in an optical cavity described by the LLE. As mentioned before, there exist two branches of CSs that coexist in the pinning region. One branch corresponds to $\mathrm{CS}$ states with an odd amount of peaks, while the other branch contains the solutions with an even amount of peaks. A typical one-peak CS is shown in Fig. 4(a). In a fiber resonator or microresonator, this
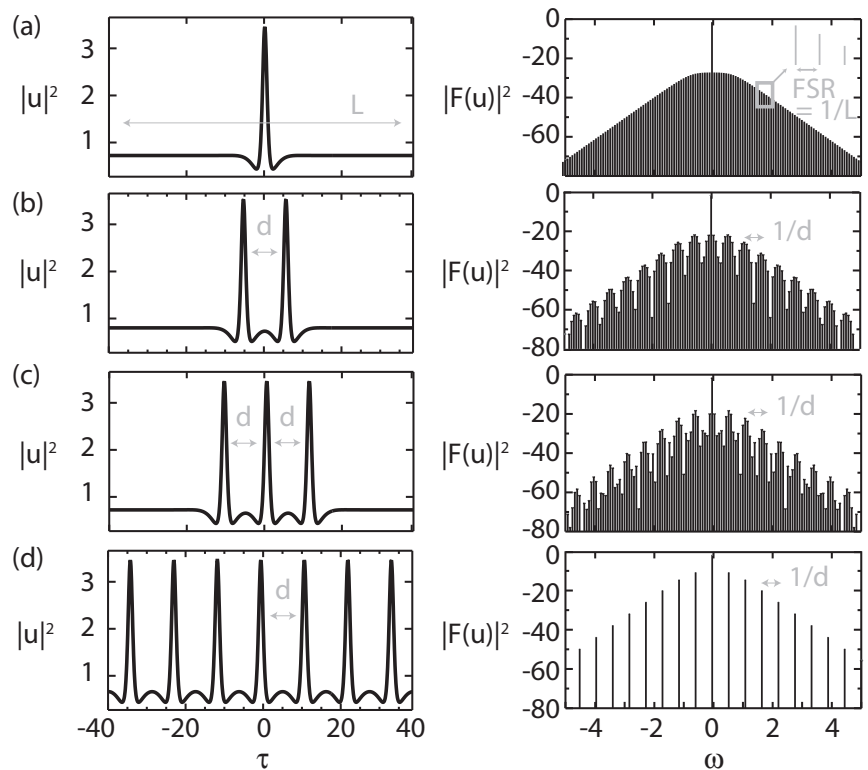

FIG. 4. Profiles of a single CS (a), two CSs (b), three CSs (c), and a pattern (d) on the left-hand side, with their corresponding frequency combs (in dB scale) on the right-hand side. The system parameters are $\theta=1.8$, the free spectral range $\mathrm{FSR}=1 / L=0.08$, and the number of discretization points was taken to be $N=1024$. 
solution would correspond to a time-localized pulse circulating in the cavity. Such a light pulse corresponds to a stable smooth frequency comb in the corresponding frequency domain, as shown in the panel on the right-hand side. The distance between all frequency modes is given by the free-spectralrange $\mathrm{FSR}=1 / L$, where $L$ is the cavity length, while the exact shape of the frequency comb is determined by the Fourier transform of the profile of the CS itself. This equivalence has also been studied in Refs. [4-6]. Solutions with two peaks and three peaks are plotted in Figs. 4(b) and 4(c), with the corresponding frequency combs. It can be seen that the effect of adding extra peaks is to introduce an extra modulation of the frequency comb. The multiple peaks of CS bound states can only coexist at well-defined separation distances $d$ between them, determined by the typical wavelength of the oscillatory tails of the CS peaks [32]. This separation distance $d$ therefore also determines the modulation distance $1 / d$ observed in the frequency comb. The modulation depth becomes more pronounced as more and more peaks are added to the solution.

In an infinite system (unbounded domain), CS peaks can be added indefinitely such that the two snaking branches in principle continue indefinitely. However, realistic systems are always bounded. In this case, the CS branches can not endlessly snake back and forth. Instead, the snaking structure is truncated when the width of the localized patterns consisting of multiple CSs approaches the domain size. In the case of fiber resonators or microresonators, periodic boundary conditions apply and the system is finite as determined by the size of the fiber loop, ring, or disk resonator. In such a finite system, the CS branches in the snaking region are generally found to connect to a branch of patterned solutions. This is also the case here, as can be seen in Fig. 2. Moreover, the branches of small amplitude CSs (i.e., the first unstable branch of single CSs) no longer bifurcate from the HSS but now bifurcate in a secondary bifurcation on the primary branch of the pattern solutions [33]. An example of a patterned solution with seven peaks is shown in Fig. 4(d). All frequency modes present in the corresponding frequency comb are now separated by $1 / d$ (or equivalently seven FSR units). Although we have used the same distance $d$ to denote the separation between various peaks in the pattern, we remark that this should not necessarily be the case, as this might vary a bit depending on the exact cavity length. We note also that CSs with $L / d-1$ peaks can be interpreted as one missing cell, or hole, in a periodic pattern.

It is clear from Fig. 2 that nonlinear optical cavities described by the LLE admit many stable and unstable localized and patterned solutions for the same set of system parameters (such as the frequency detuning and the pump power). Having such a highly multistable landscape has important consequences if one aims at creating a stable Kerr frequency comb. Every stable solution of the LLE has its own basin of attraction in the infinite-dimensional phase space. Choosing the correct initial conditions and/or writing process will thus be essential to target the structure one would like to have in the nonlinear resonator. Minor changes might result in obtaining a homogeneous solution, a single CS, a group of CSs, or a pattern, all of which correspond to different frequency combs.

In the next section, we will analyze the spatial dynamics of the homogeneous steady-state solutions as this will provide valuable information about where one can expect stable CSs to be found. We will verify this theory in Sec. V and will examine the rich temporal dynamics of CSs, which is also reflected in the corresponding frequency combs.

\section{SPATIAL DYNAMICS}

The solutions $u$ of the LLE in which we are interested, namely, CSs and localized patterns (groups of CSs), are stationary solutions of (1) (a PDE), i.e., they are such that $\partial_{t} u=0 .{ }^{1}$ Under this condition, Eq. (1) can be simplified to a special dynamical system of first-order real ODEs in which space and fast time, $\varphi$ in our case, takes the role usually played by time (see, e.g., Refs. [13,26,34,35]), namely,

$$
\begin{aligned}
& d_{\varphi} u_{1}=u_{3}, \\
& d_{\varphi} u_{2}=u_{4}, \\
& d_{\varphi} u_{3}=u_{2}+\theta u_{1}-u_{1} I_{s}, \\
& d_{\varphi} u_{4}=-u_{1}+\theta u_{2}-u_{2} I_{s}+u_{0},
\end{aligned}
$$

with $u_{1}=\operatorname{Re}(u), u_{2}=\operatorname{Im}(u), u_{3}=d_{\varphi} u_{1}, u_{4}=d_{\varphi} u_{2}$, and $I_{s}=|u|^{2}=u_{1}^{2}+u_{2}^{2}$. Bounded trajectories of this dynamical system correspond to the stationary solutions of (1). In particular, it contains all information about the stability of the stationary solutions and describes what is known as their spatial dynamics (spatial, because this technique has traditionally been applied to transverse structures). The spatial dynamics describes also how different solutions can coexist (if at all) in the cavity and how each one connects to the other, hence revealing in which parts of the parameter space particular stationary solutions can potentially exist. Clearly, this has important practical implications for Kerr frequency combs.

The fixed points of Eqs. (5) are the HSSs $u_{s}$ of the original evolution equation (1), and their stability in space $\varphi$ can be analyzed by introducing the ansatz $u=u_{s}+\epsilon e^{\lambda \varphi}$ into Eqs. (5). ${ }^{2}$ Keeping only linear terms in (small) $\epsilon$ leads to the following condition for what are known as the spatial eigenvalues $\lambda$ :

$$
\lambda^{4}+\left(4 I_{s}-2 \theta\right) \lambda^{2}+\theta^{2}-4 \theta I_{s}+3 I_{s}^{2}+1=0,
$$

as shown in the Appendix.

An important property of the spatial dynamics is reversibility, that stems from the invariance of the LLE (1) with $\partial_{t} u=0$ under the $\varphi \rightarrow-\varphi$ transformation, and, equivalently, of Eqs. (5) under the transformation $\left(u_{1}, u_{2}, u_{3}, u_{4}\right) \rightarrow$ $\left(u_{1}, u_{2},-u_{3},-u_{4}\right)$ [13]. Note that this arises in the temporal Kerr comb problem $(\varphi=\tau)$ because of our approximation to consider only second-order dispersion. Reversibility implies that the spatial eigenvalues always come in pairs (cf., e.g., [35]): each spatial eigenvalue $\lambda$ is accompanied by its counterpart $-\lambda$, and this property manifests also in that Eq. (6)

\footnotetext{
${ }^{1}$ This treatment can also be extended for moving solutions, e.g., fronts, in the reference frame that travels with the solution.

${ }^{2}$ We recall that $\varphi$ can be either the transverse spatial coordinate $x$ or the fast time $\tau$ in the case of fibers and microresonators. In this article, we will keep referring to $\varphi$ as being a spatial coordinate to avoid confusion with the cavity time $t$.
} 
TABLE I. Labels of qualitatively different spatial eigenspectra and the transitions between these regions.

\begin{tabular}{cclc}
\hline \hline Cod & $\left(\lambda_{1,2}, \lambda_{3,4}\right)$ & \multicolumn{1}{c}{ Name } & Label \\
\hline Zero & $\left( \pm q_{0} \pm i k_{0}\right)$ & Double-focus & $\mathrm{I}$ \\
Zero & $\left( \pm q_{1}, \pm q_{2}\right)$ & Double-saddle & $\mathrm{II}$ \\
Zero & $\left( \pm i k_{1}, \pm i k_{2}\right)$ & Double-center & III \\
Zero & $\left( \pm q_{0}, \pm i k_{0}\right)$ & Saddle-center & $\mathrm{IV}$ \\
One & $\left( \pm q_{0}, 0\right)$ & Rev. Takens-Bodganov & $\mathrm{SN}_{h 2}(\mathrm{RTB})$ \\
One & $\left( \pm i k_{0}, 0\right)$ & Rev. Takens-Bodganov-Hopf & $\mathrm{SN}_{h 2}(\mathrm{RTBH})$ \\
One & $\left( \pm q_{0}, \pm q_{0}\right)$ & Belyakov-Devaney & $\mathrm{BD}$ \\
One & $\left( \pm i k_{0}, \pm i k_{0}\right)$ & Hamiltonian-Hopf & $\mathrm{MI}(\mathrm{HH})$ \\
Two & $(0,0)$ & Quadruple zero & $\mathrm{QZ}$ \\
\hline \hline
\end{tabular}

is only a function of $\lambda^{2}$, not of $\lambda$ alone [Eq. (6) is a biquadratic equation]. Thus, a repelling eigenvalue is accompanied by another attracting one with the same rate [repelling (attracting) means that the spatial dynamics takes the field of the solution away (towards) the HSS $u_{s}$ ]. The fact that the characteristic equation is biquadratic can be understood more easily by performing a linear stability analysis of (1). This is shown in detail in the Appendix.

The spatial eigenvalues can be easily obtained by solving Eq. (6), that yields

$$
\lambda= \pm \sqrt{\theta-2 I_{s} \pm \sqrt{I_{s}^{2}-1}} .
$$

These eigenvalues provide all relevant information about the HSSs, and allow us to identify different regions in parameter space, in terms of the form of the leading eigenvalues, i.e., those whose real part is closer to zero. Three characteristic cases can be distinguished:

(1) The eigenvalues are organized as a quartet of complex eigenvalues $\lambda= \pm q_{0} \pm i k_{0}$, such that a trajectory leaving (and approaching) the HSS presents oscillations. This is the case corresponding to label I in Fig. 6 and Table I.

(2) The leading eigenvalues are a purely real doublet $\lambda=$ $\pm q_{0}$, such that a trajectory leaving (and approaching) the HSS is monotonic (label II in Fig. 6 and Table I).

(3) The leading eigenvalues are a purely imaginary doublet $\lambda= \pm i k_{0}$, such that the HSS is temporally unstable (labels III and IV in Fig. 6 and Table I).

Table I shows the various possibilities of how the eigenvalues can be organized and it also contains the transitions between the different regions, which are codimension-1 and -2 objects (indicated Cod in the Table. ${ }^{3}$ For a more in-depth description of all transitions and their names, we refer to Refs. [35,36]. A similar analysis of the spatial dynamics in the Lugiato-Lefever equation has recently also been reported in Ref. [37].

Figure 5 shows the organization of the spatial eigenvalues corresponding to the HSSs in the parameter plane $\left(\theta, I_{S}\right)$. In

\footnotetext{
${ }^{3}$ Codimension refers to the number of conditions that have to be specified in parameter space, and, thus, codim- 1 and -2 transitions are, respectively, lines and points in a two-dimensional parameter space.
}

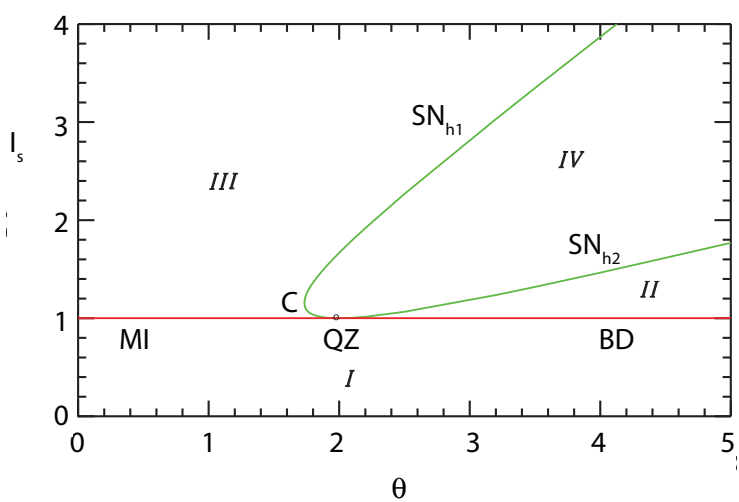

FIG. 5. (Color online) All different regions that have a qualitatively different spatial eigenspectrum are shown in the parameter plane $\left(\theta, I_{s}\right)$, as obtained from Eq. (6). The notation used is further clarified in Table I.

order to keep a closer connection to the experimental control parameters, however, we prefer plotting the structure of the spatial eigenvalues in the parameter plane $\left(\theta, u_{0}\right)$, shown in Fig. 6. A direct comparison between the different regions in Figs. 5 and 6 requires taking into account that the relationship between $I_{s}$ and $u_{0}$ is bistable in a certain range (cf. the green line in Fig. 2 for selected values of $\theta$ ). In particular, the $\mathrm{SN}_{h 2}$ transition takes place for large $u_{0}$ and small $I_{s}$, while, in turn, the $\mathrm{SN}_{h 1}$ takes place for small $u_{0}$ and large $I_{s}$. This explains that $\mathrm{SN}_{h 1}$ is above $\mathrm{SN}_{h 2}$ in Fig. 5, while $\mathrm{SN}_{h 1}$ is below $\mathrm{SN}_{h 2}$ and the $\mathrm{BD}$ line in Fig. 6. In particular, in the area between $\mathrm{SN}_{h 1}$ and $\mathrm{SN}_{h 2}$ in Fig. 6, regions I, III, and IV overlap. In the plane $\left(\theta, u_{0}\right)$, we focus mainly in the spatial eigenvalues corresponding to the lower branch HSS in the bistable region for $\theta>\sqrt{3} .^{4}$ In both figures, all labels are defined in Table I, allowing us to understand the different regions. Here below, we will discuss the different regions in Fig. 6 in more detail.

A pair of double zeros of Eq. (6) define a MI line for $\theta<2$ in parameter space (Fig. 6) given by

$$
u_{0}=\sqrt{1+(1-\theta)^{2}} \text {. }
$$

This corresponds to $I_{s}=1$ (see also Fig. 5). This degenerate pair of double zeros is given by the purely imaginary eigenvalues $\lambda= \pm i \sqrt{2-\theta}$. In dynamical systems theory, this bifurcation is known as a Hamiltonian-Hopf $(\mathrm{HH})$. The Hamiltonian-Hopf bifurcation line separates the region I where the HSS is a double-focus (has a quartet of complex eigenvalues) from region III where the HSS is a double-center with four purely imaginary spatial eigenvalues. In this region III the HSS is unstable to spatiotemporal perturbations, and patterned solutions develop.

The MI becomes a Belyakov-Devaney (BD) transition for $\theta>2$ through a quadruple zero (QZ) point at $\theta=2$, where $\lambda=0$ with multiplicity four. On the BD line, the spatial eigenvalues are given by two pairs of pure real eigenvalues $\lambda=$ $\pm \sqrt{\theta-2}$. Note that, as the MI (HH), the BD also occurs for $I_{s}=1$, although, strictly speaking, it is not a real bifurcation as $\operatorname{Re}(\lambda) \neq 0$ at the transition. The BD line separates region I from

\footnotetext{
${ }^{4}$ The upper branch HSS is always unstable in this case.
} 


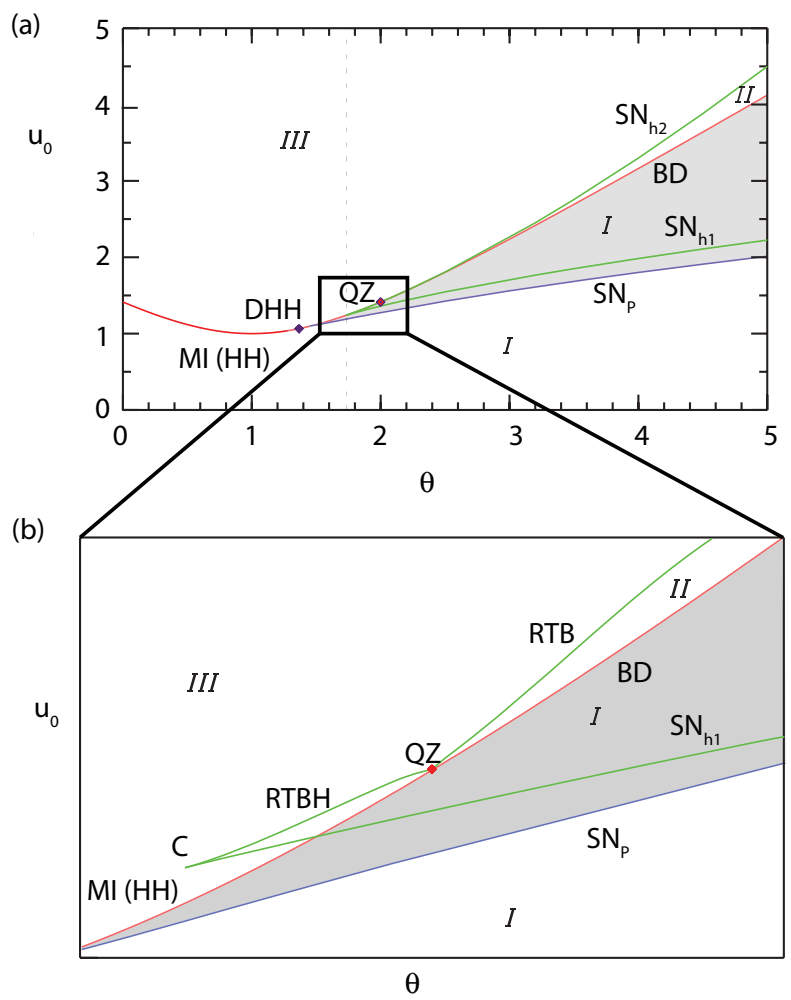

FIG. 6. (Color online) In panel (a) all different regions that have a qualitatively different spatial eigenspectrum are shown in the parameter plane $\left(\theta, u_{0}\right)$, as obtained from Eq. (6). In the bistable region between $\mathrm{SN}_{h 1}$ and $\mathrm{SN}_{h 2}, I_{s}$ in Eq. (6) is chosen such that it corresponds to the (stable) lower intensity HSS. The notation used is further clarified in Table I. $\mathrm{SN}_{P}$ denotes the saddle-node bifurcation where the (stable) pattern is born. The light gray region denotes the region where one can expect to find arbitrary sequences of CSs. In region II only single, or closely packed, CSs can be found. Panel (b) shows a closer zoom of the organization of the spatial eigenvalues around the quadruple zero (QZ) point where all eigenvalues are zero.

region II, where the HSS is a double-saddle. After crossing this line into region II the HSS does not lose its stability, and no patterned solutions develop until $\mathrm{SN}_{h 2}$ is crossed. Thus, single or closely packed CSs (not arbitrary sequences) can be found in region II.

For $\theta>2$, the $\mathrm{SN}_{h 2}$ is a reversible Takens-Bogdanov (RTB) bifurcation with two zero spatial eigenvalues and two real eigenvalues $\left( \pm q_{0}\right)$. For $\theta<2$, the $\mathrm{SN}_{h 2}$ is a reversible TakensBogdanov-Hopf (RTBH). On the RTBH line, two eigenvalues are zero and two are purely imaginary $\left( \pm i k_{0}\right)$ [see Fig. 6(b) and Table I].

In addition to the properties of HSSs, the spatial eigenvalues also explain the asymptotic behavior of the CS profile as $\varphi \rightarrow \pm \infty$. This work focuses on CSs that fall in the general class of localized structures that arise through the connection of a stable homogeneous state and a subcritical pattern [38-42]. In the context of the spatial dynamics, such localized solutions are homoclinic orbits to a fixed point (the HSS), that pass very close (arbitrarily close increasing the number of peaks of the CS) to a periodic orbit (the patterned state) (see Fig. 7). The shape of the front leaving and approaching the HSS (at the same rate, due to reversibility) is given by the leading

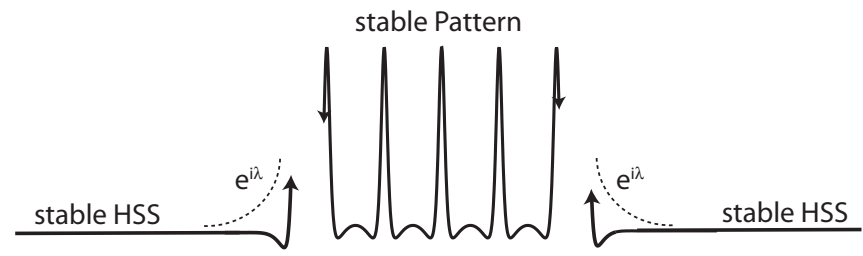

FIG. 7. Sketch of the spatial dynamics leading to CSs. A CS can be interpreted as a heteroclinic connection from a stable HSS to a stable pattern and another one from the pattern back to the HSS. The trajectory approaches the HSS according the leading spatial eigenvalues $\lambda$ of the HSS, which characterize the shape of the CS tails.

spatial eigenvalues found by solving (6). In order for (multiple) CSs to exist in any arbitrary order in the nonlinear resonator, it is required for the fronts to have oscillatory tails, so that the tails avoid the merging and annihilation of CSs $[35,36]$. Various CSs in the system are able to coexist in the cavity as they can lock to each other via their overlapping oscillatory tails, and this has been shown to determine the possible locations (and separations) of CSs in a nonlinear cavity described by the LLE [32]. Equation (6) should exhibit a complex quartet of eigenvalues in such a case.

The region where the stable homogeneous solution has a quartet of complex eigenvalues is region I. The gray region in Fig. 6 is the area where region I overlaps with the region of stable patterned solutions (above the $\mathrm{SN}_{p}$ line $^{5}$ ), such that all conditions are favorable to find CSs. Figure 8 provides more insight into how the real part $q_{0}$ and the imaginary part $k_{0}$ of the spatial eigenvalues of the stable HSS depend on the system

\footnotetext{
${ }^{5}$ The $\mathrm{SN}_{p}$ line in Fig. 6 has been computed numerically, and it starts from the degenerate Hamiltonian Hopf (DHH) point where the patterns become subcritical (see Sec. II).
}
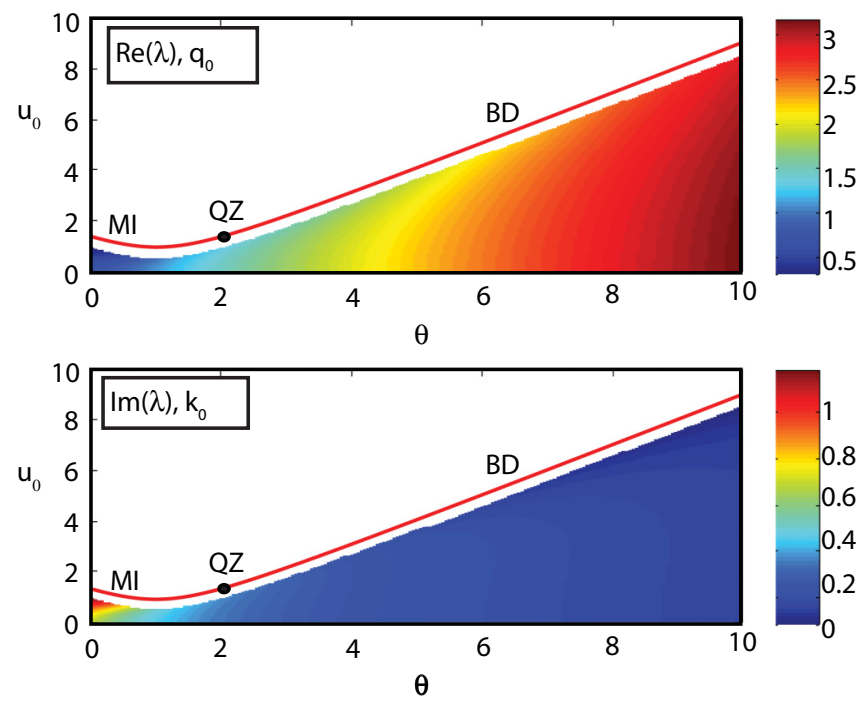

FIG. 8. (Color online) In the region I, where all spatial eigenvalues are a quartet of complex eigenvalues $\lambda_{s}= \pm q_{0} \pm i k_{0}$, we plot the real part $q_{0}$ and the imaginary part $k_{0}$ as a function of the detuning $\theta$ and the pump amplitude $u_{0}$. 


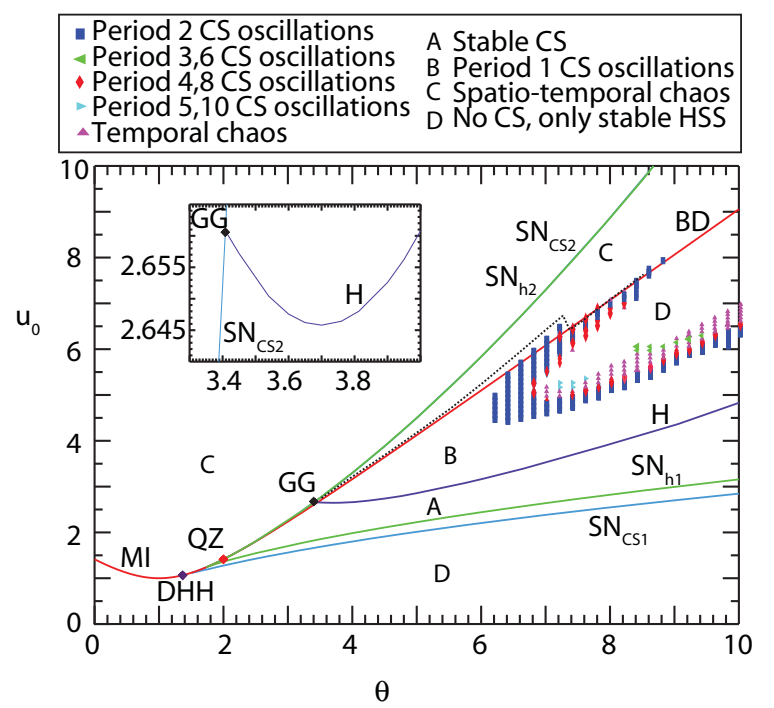

FIG. 9. (Color online) The temporal dynamics of single CSs is shown and related to the transition lines obtained from the spatial dynamics also depicted in Fig. 6. In region A, single CSs are stable. Such CSs can be destabilized by a Hopf bifurcation $H$ leading to time-oscillating CSs, found in region B. In region C, spatiotemporal chaos is found. In region D, only the stable HSS is found. Finally, more complicated time dynamics such as temporal chaos is found in the region denoted by the markers. For more information about the notation of the spatial dynamics, we refer to Table I. The inset shows a zoom of the bifurcation lines around the Gavrilov-Guckenheimer (GG) point.

parameters $\left(\theta, u_{0}\right)$. One can see that in the bistable region ${ }^{6}$ $\theta>\sqrt{3}, k_{0}$ is quite small and decreases as the detuning $\theta$ increases, while the real part $q_{0}$ strongly increases for higher $\theta$. Therefore, the oscillatory tails of a CS will be strongly damped at higher values of the detuning $\theta$.

Isolated localized structures, formed by either a single peak or a number of closely packed peaks, can also exist in region II for intracavity intensities above $I_{s}=1$ since the stable HSS still coexists with patterned solutions. In this region, however, CSs have monotonic tails and two separated CSs will move towards each other and merge.

\section{TEMPORAL DYNAMICS OF CAVITY SOLITONS}

In this section, we characterize the regions of existence of single CSs in the parameter space $\left(u_{0}, \theta\right)$ defined by the pump power and the cavity detuning. In Fig. 9, we have again plotted all characteristic lines obtained from our analysis of the spatial dynamics in the previous section (see also Fig. 6). Moreover, by combining linear stability analysis and time evolution simulations, we have characterized not only the existence of CSs, but also their temporal stability and dynamics (see also [20]). Using a Newton-Raphson method

\footnotetext{
${ }^{6} \operatorname{At} \theta=\sqrt{3}$, the system switches from being monostable to bistable. In Fig. 6, this is shown by the Cusp bifurcation (denoted by the letter C) where the two saddle-node bifurcations of the HSSs $\left(\mathrm{SN}_{h 1}\right.$ and $\mathrm{SN}_{h 2}$ ) are born.
}

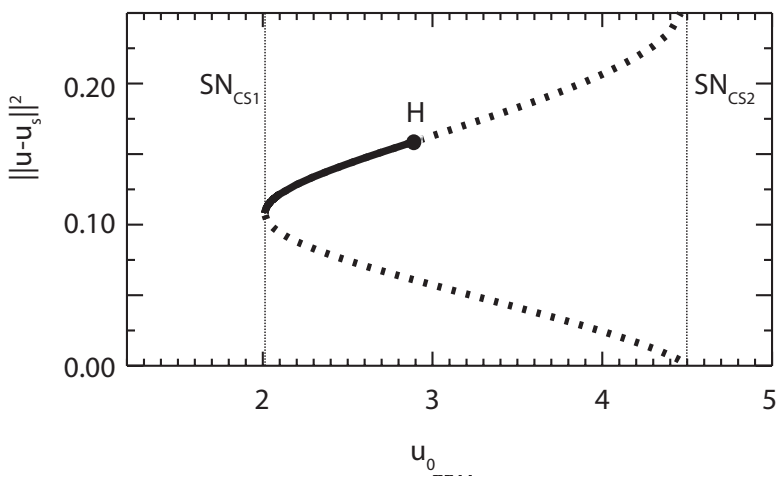

FIG. 10. Bifurcation diagram of the single peak CS, showing the presence of a Hopf instability $H$ for $\theta=5$. Solid (dashed) lines represent stable (unstable) CSs.

we can numerically track the CS solutions in parameter space and calculate their temporal stability. This allows us to accurately determine the location of the Hopf bifurcation line $H$. All other dynamical regimes have been determined numerically using time evolution simulations. In region A, stable CSs can be found. This region is delimited by the saddle-node bifurcation $\mathrm{SN}_{\mathrm{CS} 1}$ (which largely coincides with $\mathrm{SN}_{P}$ in the bistable region) and the Hopf bifurcation line $H$ where the CS is destabilized. One can notice that the region of existence of stable CSs is largely within the gray region of Fig. 6.

When crossing the Hopf bifurcation line $H$, the CS is no longer stable and displays time-periodic oscillations. This region $\mathrm{B}$ has recently also been demonstrated experimentally [20]. An example of such a Hopf instability is shown in Figs. 10 and 11. Figure 10 shows the bifurcation diagram of the single peak CS for a detuning $\theta=5$, showing the presence of a Hopf instability $H$. Crossing this Hopf bifurcation, the CS still exists and remains localized, but oscillates in time with a fixed period [see Fig. 11(a)]. The corresponding frequency comb thus also similarly oscillates in time as shown in Fig. 11(b). Examples of how both the CS profile and the frequency comb change during one oscillation period are given in Figs. 11(c) and 11(d). As the CS oscillates, the envelope of the frequency comb is modulated. Such modulation becomes more pronounced as the amplitude of the CS oscillation increases with increasing values of the pump power $u_{0}$ and detuning $\theta$. The simultaneous occurrence of a Hopf bifurcation and a saddle-node bifurcation, determined by the three temporal eigenvalues $\lambda_{a, b}= \pm i \omega$ and $\lambda_{c}=0$, with $\omega>0$, defines the codimension-2 point known as Gavrilov-Guckenheimer (GG) or Fold-Hopf bifurcation [43]. Thus, the Hopf bifurcation line emerges from the GG point, and although the Hopf line looks like it terminates perpendicularly to $\mathrm{SN}_{\mathrm{CS} 2}$, the inset in Fig. 9 shows that it in fact approaches the GG point in a tangential manner.

For higher values of the detuning, more complex oscillatory behavior can be found as well, such as period-2 oscillations, period-3 oscillations, period- $N$ oscillations, and eventually temporal chaos [20]. An example of such temporal chaos is given in Fig. 12 where one can see that the CS remains localized, but its envelope is oscillating in a chaotic way in 
(a)
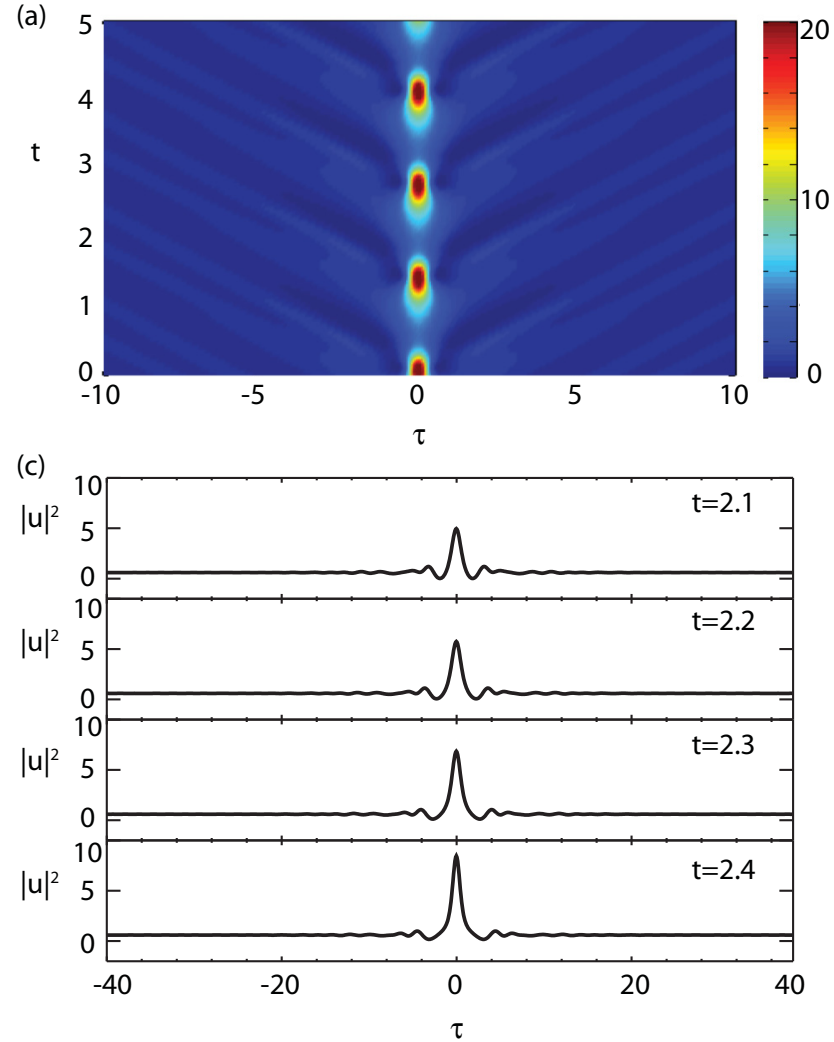

(b)

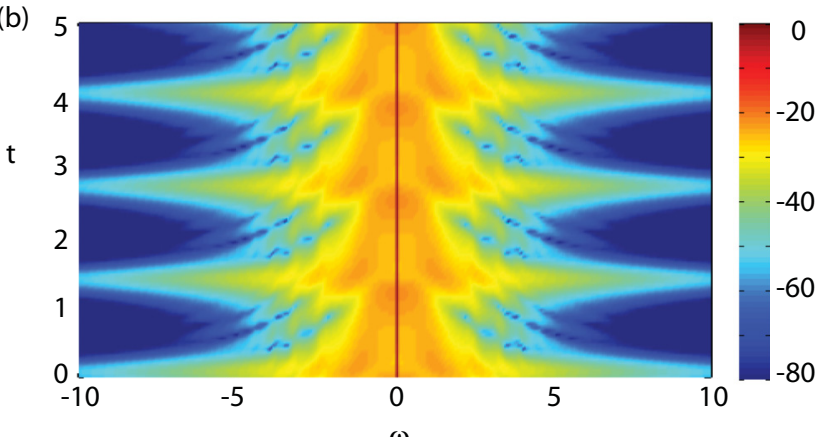

(d)

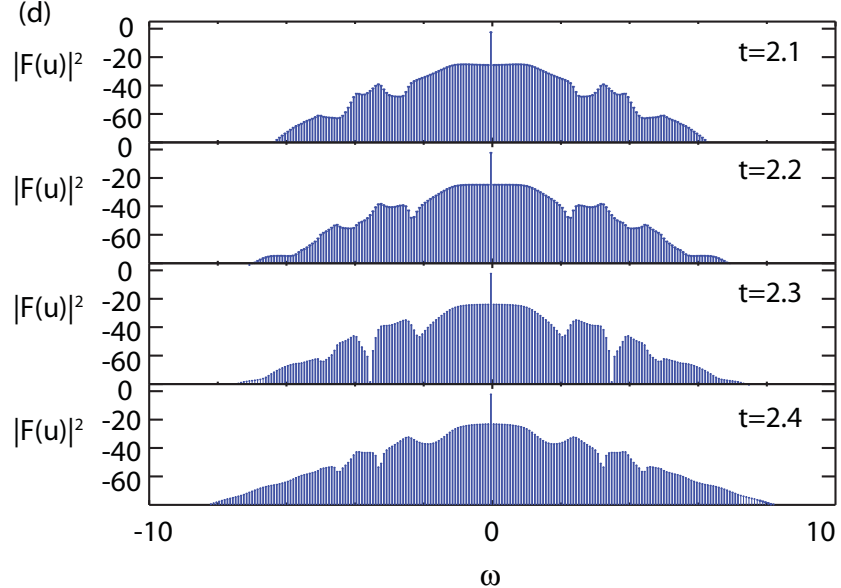

FIG. 11. (Color online) Spatiotemporal evolution of a CS and its corresponding frequency comb (in $\mathrm{dB}$ scale) is plotted for $u_{0}=3.5$ and $\theta=5$. Panels (a) and (b) show the time evolution in real and frequency space, respectively. Panels (c) and (d) show several profiles within one oscillation period of the CS, both in the time (D) and frequency domain (E).
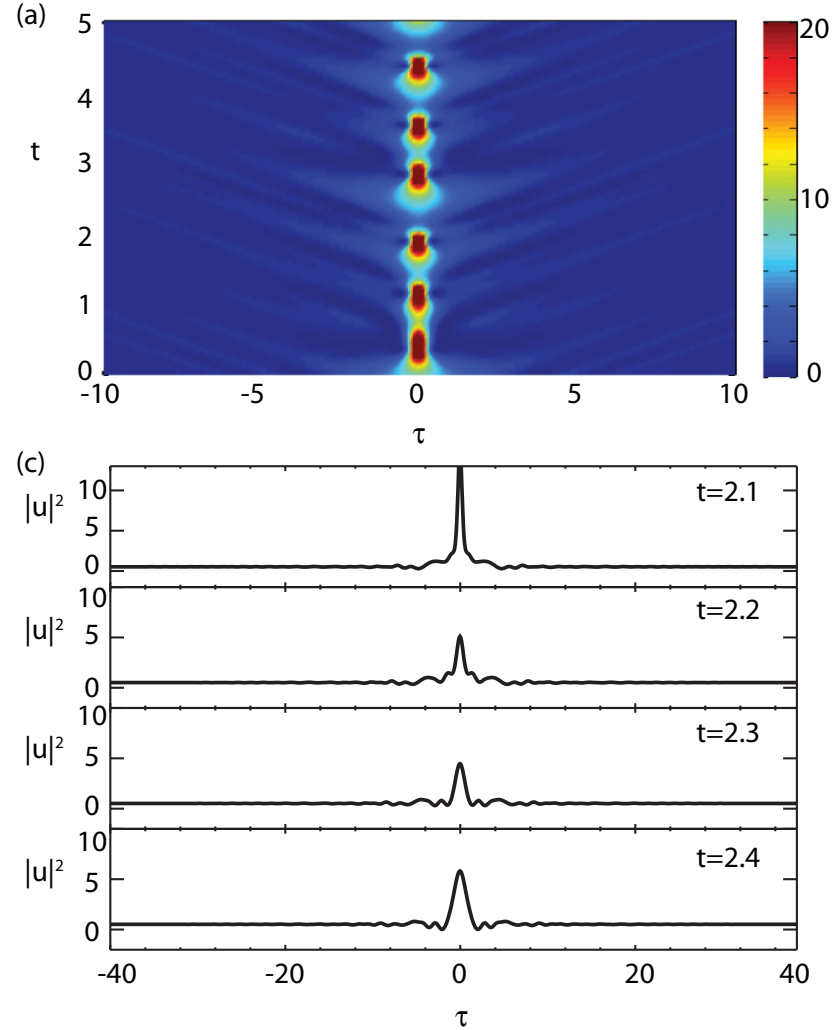
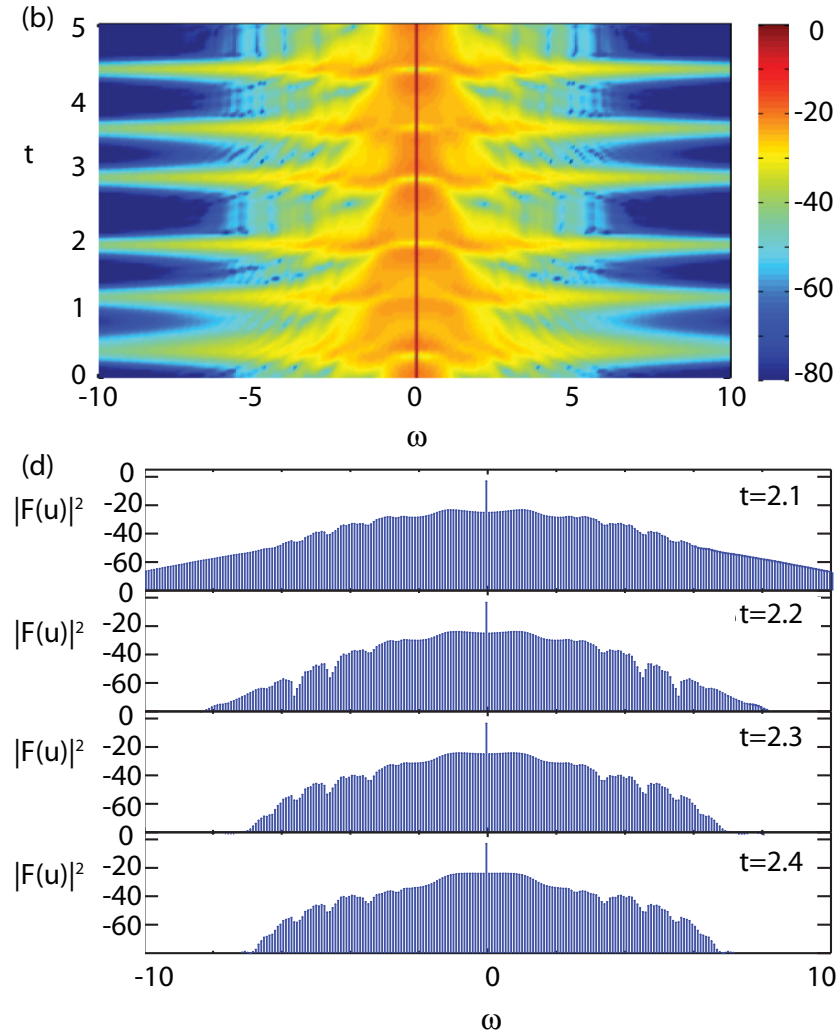

FIG. 12. (Color online) Spatiotemporal evolution of a CS and its corresponding frequency comb (in $\mathrm{dB}$ scale) is plotted for $u_{0}=6.9$ and $\theta=10$. The panels show the same as in Fig. 11, but now for temporal chaos. 

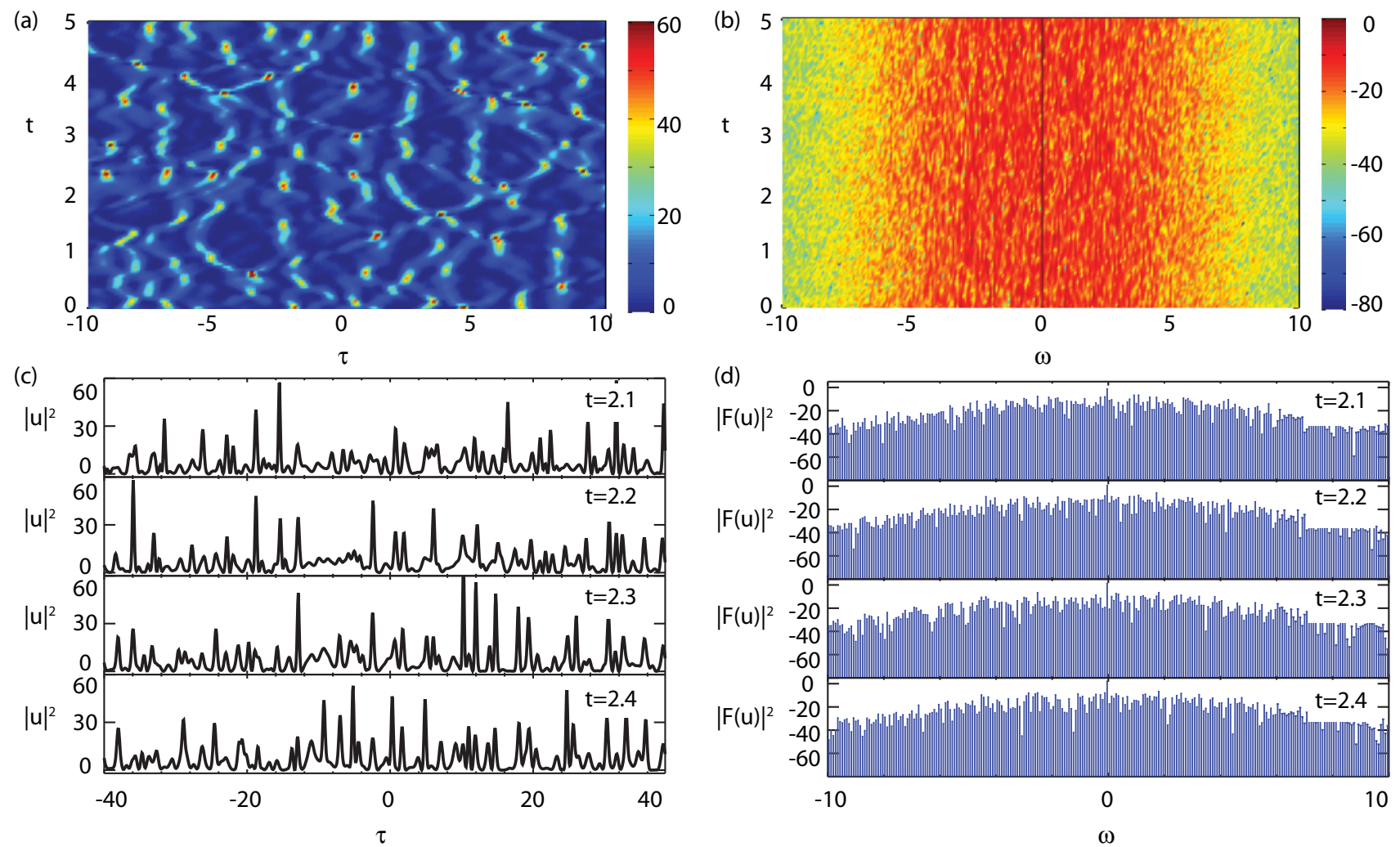

FIG. 13. (Color online) Spatiotemporal evolution of a CS and its corresponding frequency comb (in $\mathrm{dB}$ scale) is plotted for $u_{0}=8$ and $\theta=8$. The panels show the same as in Fig. 11, but now for spatiotemporal chaos.

time. Likewise, the envelope of the corresponding frequency comb will change chaotically in time. For higher values of the pump power $u_{0}$ one also encounters large regions with a different type of chaotic response, called spatiotemporal chaos or optical turbulence, as denoted by the letter $\mathrm{C}$ in Fig. 9. An example of such chaotic behavior is shown in Fig. 13, where it is clear that one can no longer distinguish clear localized CSs. Instead, the entire cavity is oscillating in a chaotic manner. The envelope of the corresponding frequency comb is no longer smooth but has significant variations of the amplitude from mode to mode due to the many different wavelengths participating in the dynamics, and it is much flatter. This regime is the one dominating the parameter space for large input powers. It can be reached directly from the HSS crossing the line $\mathrm{SN}_{h 2}$ (Fig. 9) or through the instabilities of a CS for powers around the BD line. The spatiotemporal chaotic regime coexists with the HSS for a range of input powers below $\mathrm{SN}_{h 2}$. The exact scenario that leads to such spatiotemporal chaos coming from a CS needs further characterization, but is seems to be closely related to the Belyakov-Devaney (BD) line where the oscillatory feature associated to the spatial eigenvalues of the HSS is lost and the tails of the CS approach the HSS in a monotonic manner. The dotted line separates periodically oscillating CS (region B) from spatiotemporal chaos (region C). We recall that, strictly speaking, the $\mathrm{BD}$ line is not a bifurcation and does not divide region $\mathrm{B}$. The origin of this dotted line is not fully understood, as well as the temporal chaotic behavior observed for higher values of detuning, but both might originate from the unfolding of the GG point [44].
The possible connection of these lines with the GG point will be studied in detail elsewhere.

\section{CONCLUDING REMARKS}

We have presented a comprehensive overview of the dynamics of the LLE with one transverse dimension in relation with the generation of frequency combs. Many different dynamical regimes, going from a single-peak stationary CS to spatiotemporal chaos, are available by changing the input pump and detuning as summarized in Fig. 9. On the one hand, the LLE finds applications to many different nonlinear optical cavities, and on the other hand, it is a prototypical model for the study of cavity solitons. Therefore, many of the conclusions concerning the generation of frequency combs from the different dynamical regimes are applicable to other systems displaying CSs.

Due to the fact that many different dynamical regimes are supported in the LLE, this model offers several different possibilities for the generation of frequency combs. In particular, the coexistence of multiple solutions differing only in the number of peaks circulating in the cavity offers the possibility to tune the shape of the comb without changing the parameters of the system. To target particular solutions, suitable initial conditions must be seeded in the cavity though. This observation may explain the variations in the shape of Kerr frequency combs observed in experiments with microresonators after the pump field is interrupted and restarted. 
Beyond regimes of stationary CSs, in very wide parameter range, the LLE displays dynamical regimes, going from regularly oscillating CSs to chaotic CSs and spatiotemporal chaos. Since these regimes appear for higher values of the input pump amplitude and the detuning, they have a much broader bandwidth. The amplitude of the Fourier modes is, however, not constant over time. Given the size of the parameter space associated with these solutions, one can not preclude that some reported experimental spectra have actually been obtained in this regime and, owing to the slow speed of optical spectrum analyzers, are therefore averaged over the dynamics.

\section{ACKNOWLEDGMENTS}

We thank P. Colet and F. Leo for stimulating discussions. This research was supported by the Research FoundationFlanders (FWO), by the Spanish MINECO, and FEDER under Grants FISICOS (Grant No. FIS2007-60327) and INTENSE@COSYP (Grant No. FIS2012-30634), by Comunitat Autonoma de les Illes Balears, by the Research Council of the Vrije Universiteit Brussel (VUB), and by the Belgian Science Policy Office (BelSPO) under Grant No. IAP 7-35. S. Coen also acknowledges the support of the Marsden Fund of the Royal Society of New Zealand.

\section{APPENDIX}

The characteristic equation (6) is obtained in the spatial dynamics formalism discussed in Sec. IV by performing a linear stability analysis around the HSSs of (5), that also correspond with the HSSs of Eq. (1). The linear stability of the HSS in $\varphi$ space can be found from the eigenspectrum associated with the linearization of the dynamical system around $u_{s}$, that results from solving for the eigenvalues of the Jacobian of (5) around the HSS,

$$
\left|\begin{array}{cccc}
-\lambda & 0 & 1 & 0 \\
0 & -\lambda & 0 & 1 \\
-i+\theta-2 u_{1} u_{2} & -u_{1}^{2} & -\lambda & 0 \\
-u_{2}^{2} & i+\theta-2 u_{1} u_{2} & 0 & -\lambda
\end{array}\right|=0
$$

that can be shown to yield (6).

Alternatively, one can perform a linear stability analysis directly on Eq. (1). This amounts to finding the small perturbations of the form $u=u_{s}+\epsilon \delta u$, where $\delta u \propto e^{\lambda \varphi}$. As $u$ is complex, one has to consider the corresponding complex conjugate, and considering the complex vector of perturbations $\left(\delta u, \delta u^{*}\right)$ one arrives to a complex Jacobian matrix from which the eigenvalues $\lambda$ can be obtained:

$$
\left|\begin{array}{cc}
-(1+i \theta)+2 i I_{s}+i \lambda^{2} & i u_{s} u_{s} \\
-i u_{s}^{*} u_{s}^{*} & -(1-i \theta)-2 i I_{s}-i \lambda^{2}
\end{array}\right|=0,
$$

that also yields the same characteristic equation (6), and where $I_{s}=\left|u_{s}\right|^{2}$. An advantage of this alternative deduction is that the biquadratic nature of (6) is clear by direct inspection of the $2 \times 2$ expression (A2) versus (A1), although we prefer using the spatial dynamics form (5) because it allows us to define a dynamical system from which the different dynamical transitions can be studied.
[1] S. Cundiff, J. Ye, and J. Hall, Sci. Am. 298, 74 (2008).

[2] P. Del'Haye, A. Schliesser, O. Arcizet, T. Wilken, R. Holzwarth, and T. J. Kippenberg, Nature (London) 450, 1214 (2007).

[3] T. J. Kippenberg, R. Holzwarth, and S. A. Diddams, Science 332, 555 (2011).

[4] S. Coen, H. G. Randle, T. Sylvestre, and M. Erkintalo, Opt. Lett. 38, 37 (2013).

[5] S. Coen and M. Erkintalo, Opt. Lett. 38, 1790 (2013).

[6] Y. K. Chembo and C. R. Menyuk, Phys. Rev. A 87, 053852 (2013).

[7] F. Leo, S. Coen, P. Kockaertl, S.-P. Gorza, P. Emplit, and M. Haelterman, Nat. Photonics 4, 471 (2010).

[8] L. A. Lugiato and R. Lefever, Phys. Rev. Lett. 58, 2209 (1987).

[9] A. B. Matsko, A. A. Savchenkov, W. Liang, V. S. Ilchenko, D. Seidel, and L. Maleki, Opt. Lett. 36, 2845 (2011).

[10] S. Barland, J. R. Tredicce, M. Brambilla, L. A. Lugiato, S. Balle, M. Giudici, T. Maggipinto, L. Spinelli, G. Tissoni, T. Knödl, M. Miller, and R. Jäger, Nature (London) 419, 699 (2002).

[11] J. M. McSloy, W. J. Firth, G. K. Harkness, and G.-L. Oppo, Phys. Rev. E 66, 046606 (2002).

[12] A. J. Scroggie, W. J. Firth, G. S. McDonald, M. Tlidi, R. Lefever, and L. A. Lugiato, Chaos, Solitons Fractals 4, 1323 (1994).

[13] D. Gomila, A. J. Scroggie, and W. J. Firth, Phys. D (Amsterdam) 227, 70 (2007).

[14] W. J. Firth, G. K. Harkness, A. Lord, J. M. McSloy, D. Gomila, and P. Colet, J. Opt. Soc. Am. B 19, 747 (2002).
[15] D. Gomila, A. Jacobo, M. A. Matías, and P. Colet, Phys. Rev. E 75, 026217 (2007).

[16] G. S. McDonald and W. J. Firth, J. Opt. Soc. Am. B 7, 1328 (1990).

[17] T. Herr, V. Brasch, J. D. Jost, C. Y. Wang, N. M. Kondratiev, M. L. Gorodetsky, and T. J. Kippenberg, Nature Photonics 8, 145 (2014).

[18] M. A. Foster, J. S. Levy, O. Kuzucu, K. Saha, M. Lipson, and A. L. Gaeta, Opt. Express 19, 14233 (2011).

[19] D. Turaev, A. G. Vladimirov, and S. Zelik, Phys. Rev. Lett. 108, 263906 (2012).

[20] F. Leo, L. Gelens, P. Emplit, M. Haelterman, and S. Coen, Opt. Express 21, 9180 (2013).

[21] L. Gelens, D. Gomila, G. Van der Sande, J. Danckaert, P. Colet, and M. A. Matías, Phys. Rev. A 77, 033841 (2008).

[22] F. Leo, A. Mussot, P. Kockaert, P. Emplit, M. Haelterman, and M. Taki, Phys. Rev. Lett. 110, 104103 (2013).

[23] M. Tlidi and L. Gelens, Opt. Lett. 35, 306 (2010).

[24] F. Mitschke, G. Steinmeyer, and A. Schwache, Phys. D (Amsterdam) 96, 251 (1996).

[25] D. Gomila and P. Colet, Phys. Rev. A 68, 011801 (2003).

[26] A. R. Champneys, Phys. D (Amsterdam) 112, 158 (1998).

[27] G. W. Hunt, M. A. Peletier, A. R. Champneys, P. D. Woods, M. A. Wadee, C. J. Budd, and G. J. Lord, Nonlin. Dynam. 21, 3 (2000). 
[28] J. Knobloch and T. Wagenknecht, Phys. D (Amsterdam) 206, 82 (2005).

[29] J. Burke and E. Knobloch, Phys. Rev. E 73, 056211 (2006).

[30] G. Kozyreff and S. J. Chapman, Phys. Rev. Lett. 97, 044502 (2006).

[31] S. Barbay, X. Hachair, T. Elsass, I. Sagnes, and R. Kuszelewicz, Phys. Rev. Lett. 101, 253902 (2008).

[32] G. Kozyreff and L. Gelens, Phys. Rev. A 84, 023819 (2011).

[33] A. Bergeon, J. Burke, E. Knobloch, and I. Mercader, Phys. Rev. E 78, 046201 (2008).

[34] M. Haragus and G. Iooss, Local Bifurcations, Center Manifolds, and Normal Forms in Infinite-Dimensional Dynamical Systems (Springer, Berlin, 2011).

[35] P. Colet, M. A. Matías, L. Gelens, and D. Gomila, Phys. Rev. E 89, 012914 (2014).
[36] L. Gelens, M. A. Matías, D. Gomila, T. Dorissen, and P. Colet, Phys. Rev. E 89, 012915 (2014).

[37] I. Balakireva, A. Coillet, C. Godey, and Y. K. Chembo, arXiv:1308.2542.

[38] Y. Pomeau, Phys. D (Amsterdam) 23, 3 (1986).

[39] S. Fauve and O. Thual, Phys. Rev. Lett. 64, 282 (1990).

[40] M. Tlidi, M. Taki, and T. Kolokolnikov, Chaos 17, 037101 (2007).

[41] P. D. Woods and A. R. Champneys, Phys. D (Amsterdam) 129, 147 (1999).

[42] P. Coullet, C. Riera, and C. Tresser, Phys. Rev. Lett. 84, 3069 (2000).

[43] J. Guckenheimer and P. Holmes, Nonlinear Oscillations, Dynamical Systems, and Bifurcations of Vector Fields (Springer, New York, 1983).

[44] P. Gaspard, Phys. D (Amsterdam) 62, 94 (1993). 